\title{
RETRACTS IN METRIC SPACES
}

\author{
LECH PASICKI ${ }^{1}$
}

\begin{abstract}
In this paper we define $S$-contractibility and two classes of spaces connected with this notion. A space $X$ is said to be $S$-contractible provided that $S$ is a function $S: X \times\langle 0,1\rangle \times X \ni(x, \alpha, y) \mapsto S_{x}(\alpha, y) \in X$ that is continuous in $\alpha$ and $y$, and for every $x, y \in X, S_{x}(0, y)=y, S_{x}(1, y)=x$. This notion is close to equiconnectedness, which can be defined as follows. A space $X$ is equiconnected if there exists a map $S$ such that $X$ is $S$-contractible and $S_{x}(\alpha, x)=x$ for all $x \in X$ and $\alpha \in I$ (cf. [4]). The results we obtain in the theory of retracts are close to those that are known for equiconnected spaces. Also the thickness of the neighborhood that can be retracted on a set in a metric space is estimated, which enables to prove a theorem belonging to fixed point theory.
\end{abstract}

1. We repeat the notions related to equiconnectedness [2].

Definitions. A local equiconnecting function for a space $X$ is a map $\lambda$ : $U \times I \rightarrow$ $X$, where $U$ is a neighborhood of the diagonal in $X \times X$ such that $\lambda\left(x_{0}, x_{1}, i\right)=$ $x_{i}, i=0,1$, and $\lambda(x, x, t)=x$ for every $x_{0}, x_{1}, x \in X, t \in I$.

The $\lambda$-extension of a subset $A \subset X$ is the smallest nonempty subset $\hat{A} \subset X$ (if it exists) such that $A \times \hat{A} \subset U$ and $\lambda(A \times \hat{A} \times I) \subset \hat{A}$. $A$ is $\lambda$-convex if $A=\hat{A}$.

A local equiconnecting function $\lambda$ is stable if for every neighborhood $N$ of any point $p \in X$ there exists a neighborhood $M$ such that $\hat{M} \subset N$ [3].

For $\mathcal{U}$ an open cover of $X$ and $n \geqslant 1$ let $X^{n}(\mathcal{U})=\left\{\left(x_{1}, \ldots, x_{n}\right) \in X^{n}\right.$ : $\left.\left\{x_{1}, \ldots, x_{n}\right\} \subset U \in \mathcal{Q}\right\}$ with the relative topology. Let $T^{n-1}$ denote the standard $(n-1)$ simplex in Euclidean $n$-space: $T^{n-1}=\left\{\left(t_{1}, \ldots, t_{n}\right) \in R^{n}: t_{i}>0, \Sigma t_{i}=\right.$ $1\}$.

A local convex structure for a space $X$ consists of an open cover $\mathscr{U}$ and a sequence of maps $\lambda^{n}: X^{n}(\mathscr{U}) \times T^{n-1} \rightarrow X, n>1$, such that

(i) $\lambda^{n}\left(x_{1}, \ldots, x_{n} ; t_{1}, \ldots, t_{n}\right)=\lambda^{n-1}\left(x_{1}, \ldots, \bar{x}_{m}, \ldots, x_{n} ; t_{1}, \ldots, \overline{t_{m}}, \ldots, t_{n}\right)$ if $t_{m}=0$,

(ii) for every neighborhood $N$ of any point $p \in X$ there exists a neighborhood $M$ such that $\lambda^{n}\left(M^{n} \times T^{n-1}\right) \subset N$ for all $n$ [5].

$X$ is called stably LEC if it admits a local equiconnecting function, and $X$ is LCS if it admits a local convex structure.

If such a map $\lambda$ is defined on the whole $X \times X \times I$ then $X$ is stably EC or CS respectively.

Received by the editors April 23, 1978 and, in revised form, March 21, 1979.

AMS (MOS) subject classifications (1970). Primary 54C15.

$K e y$ words and phrases. Retraction, metric space, contractible set.

${ }^{1}$ The author is indebted to the referee for his valuable remarks.

(c) 1980 American Mathematical Society 0002-9939/80/0000-0178/\$02.50 
2. Definition 1. Let a set and a function $S$ be given that satisfy the following conditions:

(1) $S: X \times I \times X \ni(x, t, y) \mapsto S_{x}(t, y) \in X$,

(2) $S_{x}(0, y)=y, S_{x}(1, y)=x$ for any $x, y \in X$.

Then for any nonempty set $A \subset X$ let $\cos A=\inf \{D \subset X: A \subset D$ and for any $\left.x \in A, t \in I, S_{x}(t, D) \subset D\right\}$. For $A=\varnothing$ let $\cos A=\varnothing$. If $\cos A=A$ then $A$ is $S$-convex.

The above definition is correct (i.e. the infimum exists) because for any two sets $E, D$ such that, for any $x \in A$ and $t \in I, S_{x}(t, D) \subset D$ and $S_{x}(t, E) \subset E$ we have $S_{x}(t, D \cap E) \subset D$ and $S_{x}(t, D \cap E) \subset E$ which implies $S_{x}(t, D \cap E) \subset D \cap E$.

Proposition 1. If $\left\{A_{s}\right\}_{s \in T}$ is a family of $S$-convex sets, then $\cap_{s \in T} A_{s}$ is $S$-convex.

Proof. Suppose that $\cap_{s \in T} A_{s} \neq \varnothing$. For any $x \in \cap_{s \in T} A_{s}, t \in I$ and $s \in T$ we have that $S_{x}\left(t, \cap_{s \in T} A_{s}\right) \subset A_{s}$ and consequently $S_{x}\left(t, \cap_{s \in T} A_{s}\right) \subset \cap_{s \in T} A_{s}$, which means that $\cap_{s \in T} A_{s}$ is $S$-convex.

Definition 2. A space is $S$-contractible if $S$ satisfies the conditions (1), (2), and, for any $x \in X,\left\{S_{x}(t, \cdot)\right\}$ is a homotopy joining the identity with a constant map (cf. [1, p. 22]).

Definition 3. A space $X$ is of $C$ type $\mathrm{I}$ if $C$ is a subset of $X$ and there exists $S$ such that $X$ is $S$-contractible and

(3) for any $x \in C$ and any neighborhood $N$ of $x$ there exists a neighborhood $U$ such that $\cos U \subset N$.

If $C=X$ then we say it is of type $I$.

Obviously any stably EC is of type $\mathrm{I}$.

Let $(M, d)$ be a metric space. For the nonempty sets $A, D \subset M$ and $r>0$ let us write $d(A, D)=\inf \{d(x, y): x \in A, y \in D\}, B(A, r)=\{x \in M: d(A, x)<r\}$ and $\operatorname{dia} A=\sup \{d(x, y): x, y \in A\}$.

TheOrem 1. Let $(M, d)$ be a metric space and let $A=\bar{A}$ be of $\partial A$ type I $(\partial A$ denotes the boundary of $A)$ such that, for any $x \notin A, d(x, A)=d(x, \partial A)$. Then $A$ is retract of $M$.

Proof. Let $\left\{U_{s}\right\}_{s \in T}$ be a locally finite open cover of $M \backslash A$ with a well-ordered family of indices $T$ and, for $\left\{a_{s}\right\}_{s \in T} \subset \partial A$, let the following condition be satisfied: if $x \in U_{s}$, then $d\left(x, a_{s}\right) \leqslant 2 d(x, A)$ for $s \in T([1, \mathrm{p} .70])$.

For $x \in M \backslash A$ let us consider $T_{x}:=\left\{s \in T: x \in U_{s}\right\}$ and let

$$
c_{s}(x)=d\left(x, M \backslash U_{s}\right) / \sup \left\{d\left(x, M \backslash U_{s}\right): s \in T_{x}\right\}
$$

and let

$$
r(x)=\left\{\begin{array}{l}
x \quad \text { for } x \in A, \\
S_{a_{s_{1}}}\left(c_{s_{1}}(x), S_{a_{s_{2}}}\left(c_{s_{2}}(x), \ldots,\left(S_{a_{s_{n}}}\left(c_{t_{n}}(x), y\right) \ldots\right)\right)\right) \text { for } x \in M \backslash A,
\end{array}\right.
$$

where $\left\{s_{1}, s_{2}, \ldots, s_{n}\right\}=T_{x}$ and $s_{1}<s_{2}<\cdots<s_{n}$ and $y \in A$. 
It is easily seen that there always exists $s \in T_{x}$ such that $c_{s}(x)=1$; then $S_{a_{s}}\left(c_{s}(x), z\right)=a_{s}$ for $z \in A$. It is trivial that $r(x) \in \operatorname{coS}\{B(x, 2 d(x, A)) \cap A\}$. So it follows from (3) that $r$ is continuous on $\partial A$. Also $r$ is continuous on $M \backslash A$ as for any $x \in M \backslash A$ there exists $B(x, \delta(x))$ which meets only finitely many $U_{s}$. Then $T_{z}$ is finite and fixed for $z \in B(x, \delta(x))$, and $r$ is a finite superposition of the same continuous maps in $B(x, \delta(x))$.

Proposition 2. Any metric space which is of type $\mathrm{I}$ is an $A R(\Re)$.

Corollary 1. Any metrizable space which is of type I is CS (cf. [5]).

DEFINITION [1, p. 219]. A compact space $X$ that is metrizable in such a way that for any $x, y \in X$ there exists exactly one $z$ such that $\rho(x, z)=\rho(y, z)=\rho(x, y) / 2$ is called a strongly convex compactum.

Corollary 2. Any strongly convex compactum is $A R$ (cf. [1, p. 219]).

Definition 4. A space $X$ is of $C$ type II provided that $C \subset X$ and there exists $S$ such that $X$ is $S$-contractible and the following condition holds:

(6) for any neighborhood $N$ of any $x \in C$ there exists a neighborhood $U$ such that for every $z \in U \cap C$ and $t \in I$ we have $S_{z}(t, U) \subset N$.

If $C=X$ let us call it type II.

It is easily seen that every type $I$ is type II.

If $X$ is a locally compact space which is $S$-contractible and $S$ is a map, and if $S_{x}(t, x)=x$ for all $x \in X$, then $X$ is of type II.

Proposition 3. Let $A=\bar{A}$ be a $\partial A$ type II subset of a metric space $(M, d)$ such that, for any $x \notin A, d(x, A)=d(x, \partial A)$ and $M \backslash A$ is finite dimensional. Then $A$ is retract of $M$.

Proof. Let $\operatorname{dim} M \backslash A \leqslant n$. Then we may assume that every $x \in M \backslash A$ belongs to at most $n+1$ sets of $\left\{U_{s}\right\}_{s \in T}$ and we follow the proof of Theorem 1. Condition (6) then ensures the continuity of $r$ on $\partial A$.

TheOREM 2. Let $A=\bar{A}$ be a $\partial$ A type II subset of a finite dimensional subspace of $a$ linear normed space $(X,\|\|)$. Then $A$ is a retract of $X$.

Proof. We construct a dense set $E$ in $\partial A$ in a special way.

$1^{\circ}$. Let $E_{1} \subset \partial A \cap B(0,1)$ be a minimal set with respect to the property that for every $x \in \partial A \cap B(0,1), d\left(x, E_{1}\right) \leqslant 1$. We denote the elements of $E_{1}$ by the natural numbers.

$2^{\circ}$. We complete $E_{1}$ to $E_{2} \subset \partial A \cap B(0,2)$ a minimal set with respect to the property that for any $x \in \partial A \cap B(0,2), d\left(x, E_{2}\right)<1 / 2$ and sign "new" points by the further numbers, etc.

$n^{\circ}$. We complete $E_{n-1}$ to $E_{n} \subset \partial A \cap B(0, n)$; for $x \in \partial A \cap B(0, n), d\left(x, E_{n}\right)<$ $1 / n$.

Now let $E=\cup_{n=1}^{\infty} E_{n}$ and for $a_{n} \in E$ let

$$
c_{n}(x)=\max \left\{0, \min \left\{1,3-d\left(x, a_{n}\right) / d(x, A)\right\}\right\}
$$


and for $x \in X, y \in A$,

$$
\begin{aligned}
& p_{1}(x, y)=S_{a_{1}}\left(c_{1}(x), y\right), \\
& p_{n}(x, y)=p_{n-1}\left(x, S_{a_{n}}\left(c_{n}(x), y\right)\right) \text { for } n>1 .
\end{aligned}
$$

We define $r: X \rightarrow A$ as follows:

$$
r(x)=\left\{\begin{array}{l}
x \text { for } x \in A, \\
\lim _{n \rightarrow \infty} p_{n}(x, y) \text { for } x \notin A .
\end{array}\right.
$$

The set $A$ is contained in a finite dimensional subspace of $X$, which with the linearity of norm yields that for $x \in B(0, r)$ each of the sets $A \cap B(x, 2 d(x, A))$ and $A \cap B(x, 3 d(x, A)) \backslash B(x, 2 d(x, A))$ contains at least one and not more than $k$ elements of the sets $E_{n(x)}$, where $n(x)=\max \{[8 / d(x, A)],[(r+1) / 6]\}$. In view of the construction of $E$ we need not consider the superposition of more than $k$ maps because there are at most $m \leqslant k$ coefficients $c_{n}(x) \in(0,1)$ before the first one that is equal to 1 . Therefore $r$ is continuous on $X \backslash A$. The continuity on $\partial A$ follows from (6).

Definition 5. A space $X$ is locally $S$-contractible if there exists $S$ satisfying (1), (2) and

(10) for any $x \in X$ there exists a neighborhood $U$ such that, for any $z \in$ $U,\left.\left\{S_{z}(t, \cdot)\right\}\right|_{U}$ is a homotopy joining the identity with a constant map.

Definition 6. A space $X$ is locally $C$ type $\mathrm{I}$ ( $C$ type II) if it is locally $S$-contractible and (3) ((6)) is satisfied.

It is obvious that every LEC space is locally $S$-contractible and every stably LEC space is locally type I.

TheORem 3. Let $A=\bar{A}$ be locally $A$ type $\mathrm{I}$ in a metric space $(M, d)$ such that, for every $x \notin A, d(x, A)=d(x, \partial A)$. Then $A$ is a retract of $D$, if $D$ is as follows.

$$
\begin{aligned}
& D=\{x \in M: \text { there exists } \varepsilon>0 \text { such that, for } y, z \\
&\left.\in \operatorname{coS}\{B(x, d(x, A)+\varepsilon) \cap \partial A\} \text { and } z \in \partial A, S_{z} \text { is a map }\right\} .
\end{aligned}
$$

Proof. Let $\varepsilon(x)=\sup \{\varepsilon:$ such that for $y, z \in \operatorname{coS}\{B(x, d(x, A)+\varepsilon) \cap \partial A\}$ and $z \in \partial A, S_{z}$ is a map $\}$ and let $\left(x_{n}\right)_{n \in N}$ be any sequence convergent in $D$, say to $x_{0}$. We have $\varlimsup_{n \rightarrow \infty} \varepsilon\left(x_{n}\right) \leqslant \varepsilon\left(x_{0}\right)$ because otherwise there would exist $n \in N$ and $\delta>0$ such that $B\left(x_{0}, d\left(x_{0}, A\right)+\varepsilon\left(x_{0}\right)+\delta\right) \subset B\left(x_{n}, d\left(x_{n}, A\right)+\varepsilon\left(x_{n}\right)\right)$. Similarly $\varepsilon\left(x_{0}\right)<\underline{\lim }_{n \rightarrow \infty} \varepsilon\left(x_{n}\right)$ because otherwise $B\left(x_{n}, d\left(x_{n}, A\right)+\varepsilon\left(x_{n}\right)+\delta\right) \subset$ $B\left(x_{0}, d\left(x_{0}, A\right)+\varepsilon\left(x_{0}\right)\right)$ would hold for a $\delta>0$. Now let $D_{\delta}=\operatorname{Int}\{x \in D: \varepsilon(x)>$ $\delta\}$ and $\mathscr{B}=\left\{B(x, \lambda(x)) \cap D_{\lambda(x)}: x \in D \backslash A\right\}$ where

$$
\lambda(x)=\min \{d(x, A), \varepsilon(x) / 4\}
$$

If for $x \in D \backslash A$ there exists $\delta>0$ such that, for each $y \in B(x, \delta), \lambda(y) \geqslant \lambda(x)$ then $x \in D_{\lambda(x)}$, otherwise a $y$ can be found such that $\lambda(y)<\lambda(x)$ (implies $x \in$ $\left.D_{\lambda(y)}\right)$ and $x \in B(y, \lambda(y))$. Hence $\mathscr{B}$ is an open cover of $D \backslash A$ and we can find a 
locally finite open cover $\left\{U_{s}\right\}_{s \in T}$ which is a star refinement of $\mathscr{B}$. If for $s \in T, x_{s}$ $\in U_{s}$, we choose $z$ for which $\operatorname{St}\left(U_{s}, \mathscr{Q}\right) \subset B(z, \lambda(z)) \cap D_{\lambda(z)}$ and $a_{s} \in$ $B\left(x_{s}, d\left(x_{s}, A\right)+\lambda(z)\right) \cap \partial A$, then for $x \in U_{s}$ we have

$$
\begin{aligned}
d\left(x, a_{s}\right) & \leqslant d\left(x, x_{s}\right)+d\left(x_{s}, a_{s}\right) \leqslant \lambda(z)+d\left(x_{s}, A\right)+\lambda(z) \\
& \leqslant 2 \lambda(z)+d\left(x_{s}, x\right)+d(x, A) \leqslant 3 \lambda(z)+d(x, A) \\
& <d(x, A)+\varepsilon(x) .
\end{aligned}
$$

Now it is easily seen that for these $\left\{U_{s}\right\}_{s \in T}$ and $\left\{a_{s}\right\}_{s \in T}$ formulas (4) and (5) give the required retraction of $D$.

Proposition 4. Any metric space which is locally type I is an ANR $(\Re)$.

Proof. In the previous considerations we put everywhere " $A$ " in place of " $\partial A$ ". We see that $D^{\prime}=\cup_{\delta>0} D_{\delta}^{\prime}$ so obtained is open. If $x \in A$ then there is $\delta>0$ for which $x \in D^{\prime}$ and hence $A \subset D^{\prime}$.

COROLlaRY. Any metrizable locally type I space is LCS (cf. [5]).

Proposition 5. Let $A=\bar{A}$ be locally $A$ type $\mathrm{I}$ in a metric space $(M, d)$ such that, for every $x \notin A, d(x, A)=d(x, \partial A)$ and $\inf \left\{\sup \left\{r: S_{z}\right.\right.$ is a map for $z, y \in$ $\operatorname{coS}\{B(x, r) \cap \partial A\}$ and $z \in \partial A\}: x \in \partial A\}=a>0$. Then $A$ is a retract of $B(A, a / 2)$.

Proof. It is enough to show that $B(A, a / 2) \subset D$, where $D$ is defined by (11). Let $x \in B(A, a / 2)$. Then $\delta(x):=a / 2-d(x, A)>0$ and

$$
\begin{aligned}
\operatorname{dia}\{B(x, d(x, A)+\delta(x) / 2) \cap A\} & \leqslant 2(d(x, A)+\delta(x) / 2) \\
= & 2(d(x, A)+a / 4-d(x, A) / 2)=d(x, A)+a / 2<a .
\end{aligned}
$$

TheOREM 4. Let $A=\bar{A}$ be a compact type I subset of a metric space $(M, d)$ and let $f: A \rightarrow M$ be a map. For each $x \in A$ and $\varepsilon>0$ let $A(f(x), \varepsilon)=$ $\operatorname{coS}\{B(f(x), d(f(x), A)+\varepsilon) \cap A\}$. Then there is an $x \in A$ such that $x \in$ $\cup_{e>0} A(f(x), \varepsilon)$ (this latter set will be denoted by $\left.A_{f(x)}\right)$.

Proof. Suppose that there exists $\delta>0$ such that, for all $x \in A, x \notin A(f(x), \delta)$. Then we take $\delta$ in place of $\varepsilon^{\prime}(x)$ and repeat the construction of $r$ from Proposition 4. The map $r \circ f: A \rightarrow A$ has a fixed point [1, p. 101] which is impossible as $(r \circ f)(x) \in A(f(x), \delta)$. Hence there exists a sequence $\left(x_{n}\right)_{n \in N}$ such that $x_{n} \in$ $A\left(f\left(x_{n}\right), \delta_{n}\right)$ with $\delta_{n} \rightarrow 0$; we may assume the sequence to converge, say to $x$. For any $\delta>0$ there exists $n_{0}$ such that for every $n \geqslant n_{0}$

$$
B\left(f\left(x_{n}\right), d\left(f\left(x_{n}\right), A\right)+\delta_{n}\right) \subset B(f(x), d(f(x), A)+\delta) .
$$

Therefore $x_{n} \in A(f(x), \delta)$ for $n \geqslant n_{0}$ and $x \in A(f(x), \delta)$. So it must be that $x \in A_{f(x)}$.

Theorem 3 and Proposition 5 have locally type I analogs; the assumption that, for $x \notin A, d(x, A)=d(x, \partial A)$ can be omitted. 


\section{REFERENCES}

1. K. Borsuk, Theory of retracts, PWN, Warszawa, 1967.

2. D. W. Curtis, Some theorems and examples on local equiconnectedness and its specializations, Fund. Math. 72 (1971), 101-113.

3. J. Dugundji, Locally equiconnected spaces and absolute neighborhood retracts, Fund. Math. 57 (1965), 187-193.

4. R. H. Fox, On fiber spaces. II, Bull. Amer. Math. Soc. 49 (1943), 733-735.

5. C.J. Himmelberg, Some theorems on equiconnected and locally equiconnected spaces, Trans. Amer. Math. Soc. 115 (1965), 43-53.

Science School of Mining and Metallurgy, Institute of Mathematics, Kraków, Al. Mickiewicza 30, Poland 